\title{
Latent ion tracks in amorphous silicon
}

\author{
T. Bierschenk, ${ }^{1, *}$ R. Giulian, ${ }^{1, \dagger}$ B. Afra, ${ }^{1}$ M. D. Rodriguez, ${ }^{1}$ D. Schauries, ${ }^{1}$ S. Mudie, ${ }^{2}$ O. H. Pakarinen,,${ }^{3,4}$ F. Djurabekova, ${ }^{4}$ \\ K. Nordlund, ${ }^{4}$ O. Osmani, ${ }^{5,6}$ N. Medvedev, ${ }^{7}$ B. Rethfeld, ${ }^{6}$ M. C. Ridgway, ${ }^{1}$ and P. Kluth ${ }^{1}$ \\ ${ }^{1}$ Research School of Physics and Engineering, Australian National University, Canberra, ACT 0200, Australia \\ ${ }^{2}$ Australian Synchrotron, Clayton, Victoria 3168, Australia \\ ${ }^{3}$ Materials Science and Technology Division, Oak Ridge National Laboratory, Oak Ridge, Tennessee 37831, USA \\ ${ }^{4}$ Helsinki Institute of Physics and Department of Physics, University of Helsinki, P.O. Box 43, 00014 Helsinki, Finland \\ ${ }^{5}$ Fakultät für Physik, Universitt Duisburg-Essen, 47057 Duisburg, Germany \\ ${ }^{6}$ Department of Physics and OPTIMAS Research Center, Technical University of Kaiserslautern, 67653 Kaiserslautern, Germany \\ ${ }^{7}$ Center for Free-Electron Laser Science at DESY, Notkestrasse 85, D-22607 Hamburg, Germany \\ (Received 23 June 2013; revised manuscript received 1 September 2013; published 25 November 2013)
}

\begin{abstract}
We present experimental evidence for the formation of ion tracks in amorphous Si induced by swift heavy-ion irradiation. An underlying core-shell structure consistent with remnants of a high-density liquid structure was revealed by small-angle $\mathrm{x}$-ray scattering and molecular dynamics simulations. Ion track dimensions differ for as-implanted and relaxed $\mathrm{Si}$ as attributed to different microstructures and melting temperatures. The identification and characterization of ion tracks in amorphous Si yields new insight into mechanisms of damage formation due to swift heavy-ion irradiation in amorphous semiconductors.
\end{abstract}

DOI: 10.1103/PhysRevB.88.174111

PACS number(s): 61.80.-x, 61.05.cf, 61.43.Bn, 61.72.uf

\section{INTRODUCTION}

Inelastic interactions of projectile ions and target electrons dominate the energy loss for swift heavy ions (SHIs) and result in intense electronic excitation which can produce a narrow region of permanent damage along the ion trajectory, a so-called ion track. The later stages of ion track formation, after the initial electronic excitation, are well described by a thermal spike mechanism., Within this formalism, the materialspecific electron-phonon coupling governs the transfer of the deposited energy to the target atoms, which induces a local increase in the lattice temperature. For sufficiently high electronic energy deposition, the melting temperature of the substrate can be exceeded, and a molten zone is formed along the ion trajectory. After rapid resolidification, a defective cylindrical region of high aspect ratio, an ion track, remains.

Ion track formation in amorphous semiconductors has long been postulated. Hedler et al. ${ }^{3,4}$ invoked ion track formation in amorphous $\mathrm{Si}(\mathrm{a}-\mathrm{Si}$ ) in support of their claim for liquid-Si polymorphism, specifically the existence of a low-density liquid (LDL) Si phase in addition to the well-known highdensity liquid (HDL) Si phase. Resolidification of LDL Si to low-density amorphous (LDA) Si then represents a glass transition for elemental Si. Under SHI irradiation, LDA Si was observed to deform plastically, ${ }^{3}$ as consistent with the ion-hammering mechanism, and was attributed to ion track formation. Indirect evidence of ion track formation in a-Si has thus been reported. Crystalline $\mathrm{Si}$ (c-Si), however, is much less susceptible to radiation damage from SHIs and requires much higher values of electronic energy deposition $S_{e}$ for damage formation. Ion track formation, for example, was only reported for irradiation with fullerene molecular ions with a threshold of $S_{e}=37 \mathrm{keV} / \mathrm{nm},{ }^{5-7}$ whereas no signature of ion tracks was observed for irradiations with single SHIs. Mixing experiments of a Ni-Si interface under irradiation ${ }^{8}$ with single SHIs suggest that melting does occur in c-Si for lower values of $S_{e}$. The molten ion track, however, appears to fully recrystallize upon cooling, indicating that melting cannot be the only criterion for ion track formation in c-Si. Ion track formation in polycrystalline $\mathrm{Si}$ has been reported by Furuno et $a l . ;^{9}$ however, the irradiation was performed on 5-nm thin evaporated layers, and the nature of the ion tracks was not determined.

While Hedler et al $^{3,4}$ postulated an ion track radius of $\approx 3 \mathrm{~nm}$ in a-Si at $14.6 \mathrm{keV} / \mathrm{nm}$ (their experimentally determined threshold for plastic deformation), experimental verification of ion track formation in $\mathrm{a}-\mathrm{Si}$ has been lacking due to the difficulties in detection caused by the lack of contrast between the amorphous ion track and the surrounding amorphous matrix. We have recently identified and characterized ion tracks in amorphous metals, ${ }^{10}$ silica $\left(\mathrm{a}-\mathrm{SiO}_{2}\right),{ }^{11,12}$ and $\mathrm{Ge}(\mathrm{a}-\mathrm{Ge})^{13}$ by utilizing synchrotron-based small-angle $\mathrm{x}$-ray scattering (SAXS). In the present study we provide direct and definitive experimental evidence for ion tracks in a-Si by combining SAXS with molecular dynamics (MD) simulations. Our theoretical approach uses Monte Carlo twotemperature model (MC-TTM) calculations as input for the MD simulations, yielding a full atomistic model of ion track formation without the need to assume melting as a criterion. The results reveal an underlying core-shell morphology and suggest, overall, that an ion track in a-Si is of greater density than the unirradiated matrix.

\section{EXPERIMENTAL DETAILS}

A $1.4-\mu \mathrm{m}$ thin surface layer of bulk c-Si(100) was rendered amorphous by ion implantation of Ge ions at multiple energies between 80 and $1450 \mathrm{keV}$. With an average Ge concentration of $1.7 \times 10^{19} \mathrm{~cm}^{-3}$, compositional changes due to the $\mathrm{Ge}$ implantation can be neglected. To investigate the difference in ion track formation between as-implanted and relaxed a-Si, selected samples were annealed at $450{ }^{\circ} \mathrm{C}$ for $30 \mathrm{~min}$. Annealing at this temperature yields a reduction of point defects and dangling bonds (relaxation) but not recrystallization. ${ }^{14}$

The samples were irradiated with $\mathrm{Au}$ ions at 89 and $185 \mathrm{MeV}$ at the Australian National University (ANU) 
TABLE I. Irradiation conditions and results from the SAXS analysis. The electronic energy deposition $S_{e}$ was calculated by the SRIM2008 code $^{15}$ and averaged over the 1.4- $\mu \mathrm{m}$ thin a-Si layer (the error is the standard deviation of $S_{e}$ within the layer).

\begin{tabular}{lccccrr}
\hline \hline a-Si & $E(\mathrm{MeV})$ & $S_{e}(\mathrm{keV} / \mathrm{nm})$ & $R_{c}(\mathrm{~nm})$ & $T_{s}(\mathrm{~nm})$ & $R(\mathrm{~nm})$ & $\rho_{s} / \rho_{c}$ \\
\hline As-implanted & 89 & $10.6 \pm 0.4$ & $2.3 \pm 0.1$ & $5.0 \pm 0.3$ & $7.3 \pm 0.4$ & $-0.07 \pm 0.02$ \\
& 185 & $15.6 \pm 0.2$ & $2.5 \pm 0.1$ & $5.4 \pm 0.3$ & $7.9 \pm 0.4$ & $-0.06 \pm 0.02$ \\
Relaxed & 185 & $15.6 \pm 0.2$ & $1.8 \pm 0.1$ & $3.7 \pm 0.3$ & $5.5 \pm 0.3$ & $-0.07 \pm 0.02$ \\
\hline \hline
\end{tabular}

Heavy-Ion Accelerator Facility. Irradiation was performed at room temperature under normal incidence with fluences ranging from $6 \times 10^{10}$ to $5 \times 10^{11}$ ions $/ \mathrm{cm}^{2}$. The electronic energy deposition in a-Si, as calculated using SRIM2008, ${ }^{15}$ is listed in Table I. The variation of $S_{e}$ over the thin layer was $\lesssim 3 \%$.

SAXS measurements were performed at the SAXS/WAXS beam line of the Australian Synchrotron using camera lengths between 968 and $7327 \mathrm{~mm}$ and an x-ray energy of $12 \mathrm{keV}$. Before the measurements, the majority of the c-Si substrate was removed by mechanically polishing to a thickness of $\approx 25 \mu \mathrm{m}$ to reduce parasitic $\mathrm{x}$-ray scattering from the substrate. Under the given irradiation conditions, no ion tracks were observed in a c-Si sample without preamorphization (not shown) consistent with reported threshold values of $S_{e}$ required for ion track formation in this phase. ${ }^{6,8}$ No ion tracks were thus present in the $\mathrm{c}-\mathrm{Si}$ substrate beneath the a-Si layer. Measurements were performed either with the sample surface aligned normal to the $\mathrm{x}$-ray beam, i.e., parallel to the ion tracks, or under an angle of $10^{\circ}$. SAXS images for these geometries are shown in Figs. 1(a) and 1(b), respectively. The straight lines in Fig. 1(a) and the short dashes in Fig. 1(b), which can also be observed in unirradiated samples, indicate parasitic scattering unrelated to ion tracks. When the sample surface is aligned perpendicular to the x-ray beam, isotropic scattering can be observed, as shown in Fig. 1(a), which is consistent with ion tracks aligned normal to the sample surface. In Fig. 1(b) narrow curved streaks are apparent in the image, reflecting the high aspect ratio of the ion tracks (several nanometers in width but micrometers in length) $)^{11}$ when the sample is tilted. The isotropic scattering and the anisotropic streaks contain identical information on the radial ion track dimensions. The width of the streaks in Fig. 1(b) at a given angle is related to the length of the ion tracks but also depends on stresses in the sample as well as deviations from

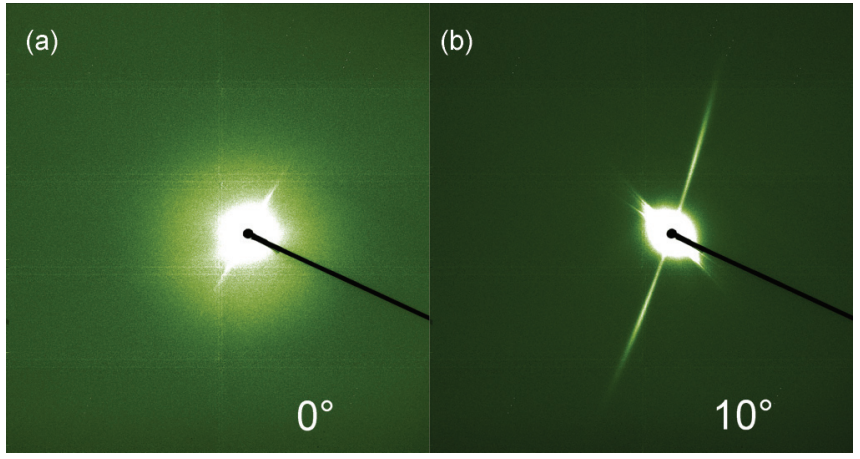

FIG. 1. (Color online) Scattering images for ion tracks in asimplanted a-Si (a) aligned and (b) tilted with respect to the incoming $\mathrm{X}$-ray beam. a perfectly parallel alignment of ion tracks. Discontinuous ion tracks with individual fragment lengths up to 50 times the ion track diameter would cause a drastic broadening of the streaks under the given angle. No significant broadening of the streaks was observed for all samples used in this work, from which we conclude ion track fragmentation was not present.

\section{RESULTS AND DISCUSSION}

\section{A. As-implanted a-Si}

Scattering spectra of as-implanted a-Si irradiated with 185$\mathrm{MeV} \mathrm{Au}$ ions are depicted in Fig. 2. The scattering intensities shown in the spectra were extracted from the streaks of the scattering images [Fig. 1(b)] by applying a narrow mask along the streaks, angularly integrating over areas of constant $q$ value within the mask and normalizing to the width of the mask. The scattering intensity scales approximately with the irradiation fluence, i.e., the number of scattering objects, confirming the absence of interparticle scattering as expected from randomly distributed well-separated ion tracks. ${ }^{11}$ Fits (solid lines in Fig. 2), assuming a cylindrical core-shell structure for the ion tracks, are in excellent agreement with the measured SAXS spectra. The scattering amplitude for this geometry is given by $^{12}$

$$
f_{1}(q)=\frac{2 \pi L \rho_{c}}{q}\left[\left(1-\frac{\rho_{s}}{\rho_{c}}\right) R_{c} \mathrm{~J}_{1}\left(R_{c} q\right)+\frac{\rho_{s}}{\rho_{c}} R \mathrm{~J}_{1}(R q)\right],
$$

with the ion track length $L$, the scattering contrasts (density change) with respect to the matrix material $\rho_{c}$ and $\rho_{s}$ of core

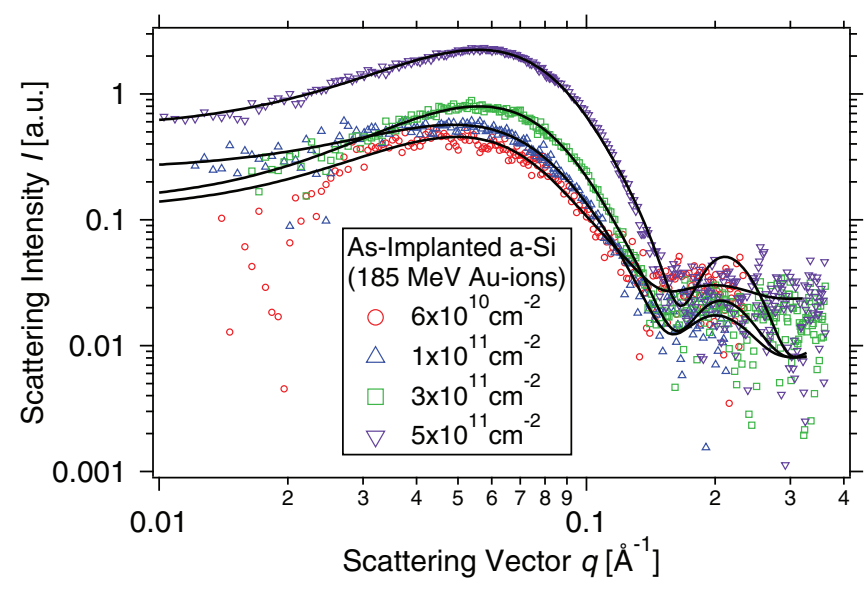

FIG. 2. (Color online) SAXS spectra (background subtracted) of ion tracks in as-implanted a-Si irradiated with $185-\mathrm{MeV} \mathrm{Au}$ ions. Solid lines are fits using a cylindrical core-shell model [Eq. (1)]. 


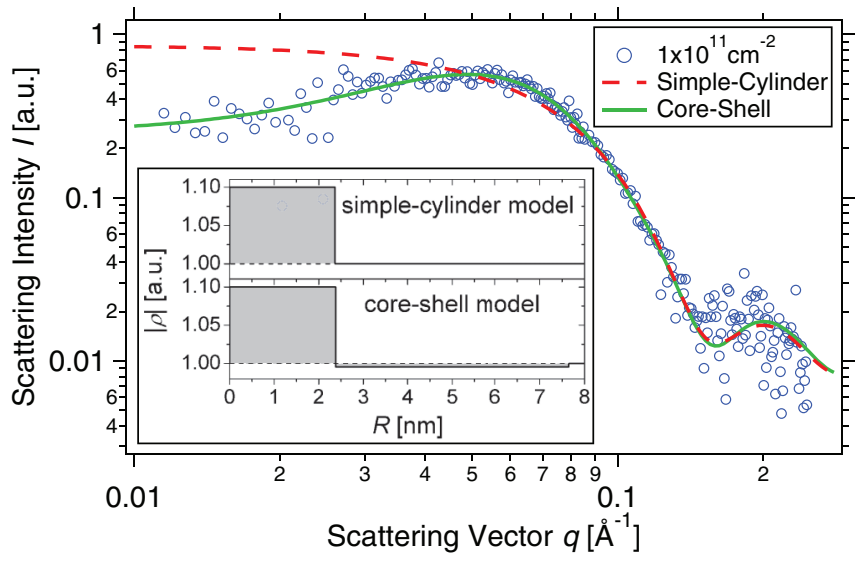

FIG. 3. (Color online) Comparison of simple [dashed line, Eq. (2)] and core-shell [solid line, Eq. (1)] cylinder models in the case of as-implanted a-Si irradiated with $185-\mathrm{MeV} \mathrm{Au}$ ions $\left(1 \times 10^{11}\right.$ ions $\left./ \mathrm{cm}^{2}\right)$. The inset shows the magnitude of the model radial density distribution function $|\rho|$, scaled to the fit parameters for both models. $|\rho|=1$ represents the density of the surrounding a-Si matrix.

and shell, respectively, the total ion track radius $R=R_{c}+T_{s}$ (with core radius $R_{c}$ and shell thickness $T_{s}$ ), and the firstorder Bessel function $\mathrm{J}_{1}$. Similar to our previous studies ${ }^{12}$, a narrow Gaussian distribution of the radius (with a standard deviation of $<10 \%$ ) was used to account for slight variations of the ion track radius and any deviation from the parallel alignment of the ion tracks. When averaged over different samples irradiated under the same conditions and measured in separate experiments, ion tracks in as-implanted a-Si show an overall radius of $7.9 \pm 0.4 \mathrm{~nm}$ composed of a $2.5 \pm 0.1 \mathrm{~nm}$ core radius and $5.4 \pm 0.3 \mathrm{~nm}$ shell thickness (see Table I). Based on the ratio of the relative scattering contrast of core and shell $\frac{\rho_{s}}{\rho_{c}}=-0.07 \pm 0.02$, the density change for the core compared to the surrounding a-Si matrix is approximately 15 times larger than for the shell. As a consequence of the negative sign of $\frac{\rho_{s}}{\rho_{c}}$, either the core or shell must be overdense and its counterpart underdense with respect to the a-Si matrix. The radial density of the ion tracks, based on the fit parameters, is shown in the inset of Fig. 3. We note that the core is the dominant feature of the ion track and its dimensions are in good agreement with the 3-nm ion track radius predicted from the ion hammering experiments for similar $S_{e}$ values ${ }^{3}$. To validate our use of a core-shell model, a comparison with a simplecylinder model with a constant density was performed. The scattering amplitude of a simple cylinder is given by ${ }^{16}$

$$
f_{2}(q)=\frac{2 \pi L R \rho_{0}}{q} \mathrm{~J}_{1}(R q)
$$

where $R$ is the ion track radius and $\rho_{0}$ is the density contrast. Fits of both model functions, the core-shell [Eq. (1)] and the simple cylinder [Eq. (2)], are compared in Fig. 3, with the inset showing the radial density distribution for the two models scaled according to the fit parameters. The dimension of the ion track in the simple-cylinder model is in good agreement with the core dimensions of the core-shell model. In contrast to the core-shell model, however, the simple-cylinder model cannot reproduce the low scattering intensity for $q \lesssim 0.04 \AA^{-1}$,

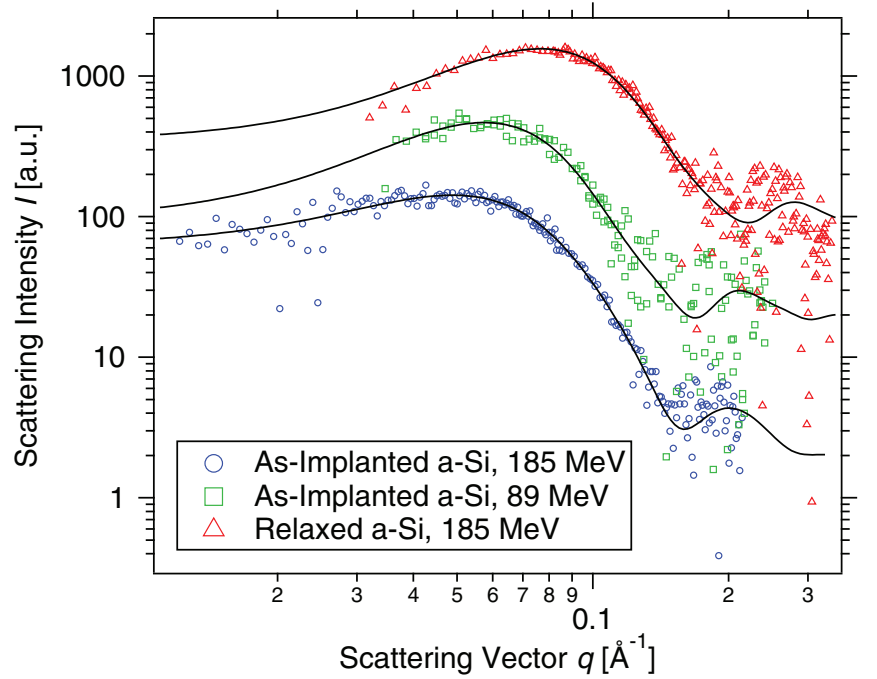

FIG. 4. (Color online) Comparison of SAXS spectra of as-implanted a-Si irradiated at 185 and $89 \mathrm{MeV}$ (both $1 \times$ $10^{11}$ ions $\left./ \mathrm{cm}^{2}\right)$ and relaxed a-Si irradiated at $185 \mathrm{MeV}(3 \times$ $10^{11}$ ions $/ \mathrm{cm}^{2}$ ). Solid lines are fits using a core-shell model. Spectra are offset for clarity.

demonstrating the core-shell model is the most appropriate of the two.

Ion tracks in as-implanted a-Si were also observed for irradiation with 89-MeV Au ions (see Fig. 4). At the lower irradiation energy, a core-shell structure is retained, but the radius is slightly reduced, which is consistent with the reduced electronic energy deposition $(10.6 \mathrm{keV} / \mathrm{nm})$ at $89 \mathrm{MeV}$. An accurate extrapolation to a threshold of $S_{e}$ for ion track formation in a-Si requires a larger data set given the nonlinear relationship between ion track radii and electronic energy deposition demonstrated in other materials, e.g., $\mathrm{a}_{-} \mathrm{SiO}_{2} \cdot{ }^{12}$ Nonetheless, the value of $10.6 \mathrm{keV} / \mathrm{nm}$ represents an upper limit for the threshold of $S_{e}$ and is less than that reported for ion hammering and plastic deformation. ${ }^{3}$ This suggests ion track formation may not be the only process operative during ion hammering.

\section{B. Relaxed a-Si}

The core-shell structure can also be observed for ion tracks in relaxed a-Si with an ion track radius $\approx 30 \%$ less than in asimplanted material (see Fig. 4 and Table I). Ion-implanted a-Si in a relaxed state has no measurable difference in mass density compared to as-implanted a-Si; however, during relaxation short-range ordering occurs where defects and dangling bonds are reduced in number, and the material moves structurally closer to an ideal continuous random network. ${ }^{14,17,18}$ In addition to structural differences, relaxation yields an $\approx 150 \mathrm{~K}$ increase in melting temperature relative to that of as-implanted a-Si. ${ }^{19,20}$ If the measured ion track diameter is representative of the maximum width of the molten zone along the ion trajectory, the increase in melting temperature for relaxed a-Si necessarily yields a decrease in ion track diameter. Although the widths of the molten cylinders in as-implanted and relaxed a-Si are of different diameter, we anticipate the mechanism for ion track formation in the these two subtly different forms of a-Si will be the same. 


\section{MD SIMULATIONS}

The scattering intensity measured with SAXS is proportional to the square of the scattering amplitude, and thus the magnitude but not the sign of the density profile is accessible. Thus both scenarios, an underdense core with an overdense shell and vice versa, must be considered. Further insight into the radial density distribution was obtained by performing MD simulations using the PARCAS code ${ }^{21}$ and Tersoff III potential, ${ }^{22}$ which produces a reasonable description of the solid-liquid-solid phase transformations in a-Si. The initial a-Si was created from c-Si using the well-established Monte Carlo approach of Wooten, Winer, and Weaire ${ }^{23}$ in a cell of dimensions $29.5 \times 29.5 \times 19.3 \mathrm{~nm}^{3}$. MC-TTM calculations describing the time-dependent energy transfer between the electronic and lattice subsystems were used as input for the MD simulations. The energy deposition that was previously used for a-Ge $\mathrm{G}^{13}$ was scaled according to the different electronic energy depositions in $\mathrm{Ge}$ and $\mathrm{Si}$ of 22 and $15.6 \mathrm{keV} / \mathrm{nm}$, respectively, as obtained by SRIM2008, ${ }^{15}$ leading to an energy of $6.02 \mathrm{keV} / \mathrm{nm}$ deposited in the MD cell. To mimic heat conduction farther into the surrounding cooler material, 0.5-nm-thick boundary regions in the $x$ and $y$ directions were maintained at $300 \mathrm{~K}$ by Berendsen temperature control. ${ }^{24}$ Immediately after deposition, there is a rapid nonequilibrium expansion, leading to a wide region of more than $9 \mathrm{~nm}$ in diameter of somewhat underdense Si. At 3 to 5 ps, a phase transition to overdense Si occurs, with a density about $3 \%$ higher than the unirradiated material. This agrees well with the measured phase transition time delay of about 5 ps reported by Beye et al. ${ }^{25}$ The cooling stage that follows lowers the density difference continuously to about $1 \%$ in about $50 \mathrm{ps}$ and defines the outer radius of the underdense region more sharply during the remaining $150 \mathrm{ps}$ of simulation time.

The MD result for the radial density distribution across the ion track at a thermally stable state 200 ps after the initial energy deposition is shown in Fig. 5. With a relative density of one corresponding to that of the initial a-Si cell, a distinct core-shell structure with an overdense core extending to $\approx 4 \mathrm{~nm}$ and a slightly underdense shell extending to $\approx 9 \mathrm{~nm}$ is clear. The radial density distribution derived from the SAXS measurements is also included in Fig. 5, and qualitative agreement with the MD simulation is readily apparent. In contrast, the use of the Stillinger-Weber potential ${ }^{26}$ in the MD simulations led to a more pronounced core-shell structure, which can be attributed to the less realistic description of the phase transitions.

The inset of Fig. 5 shows the radial MD temperature profile. Assuming only the melting criterion, the ion track radius for the different melting temperatures of as-implanted and relaxed a-Si ${ }^{19,20}$ can be estimated to $\approx 8 \%$. The observed $30 \%$ change is thus a likely consequence of microstructural differences in addition to differences in only the melting temperature.

Given the volumes of core and shell and their relative densities, the ion track as a whole is of greater density than the unirradiated matrix. We suggest the overdense ion track core could conceivably contain frozen-in structural remnants of the molten HDL Si phase, potentially in the form of the pressureinduced high-density amorphous (HDA) Si phase reported by McMillan et al. ${ }^{27}$ The rapid cooling rate associated with

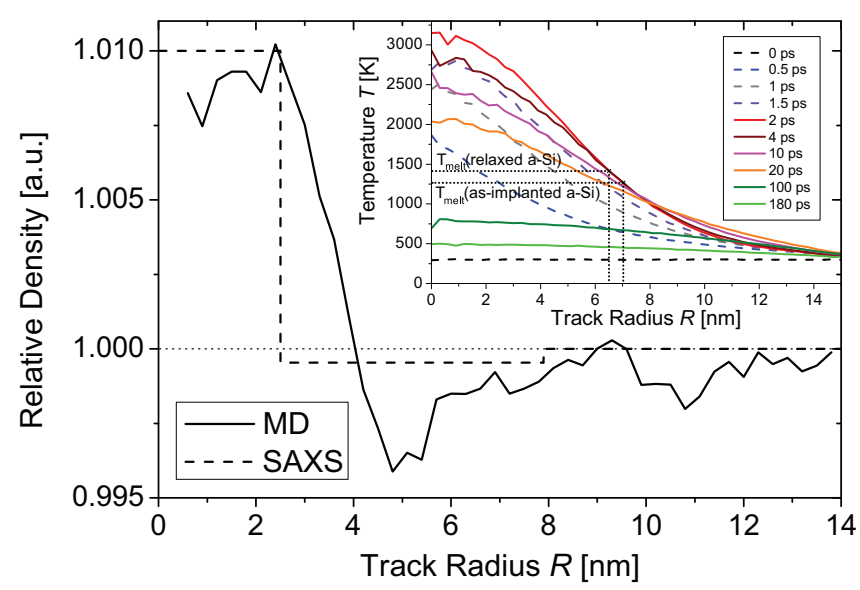

FIG. 5. (Color online) Ion track density profiles of MD simulation (five-point average, $200 \mathrm{ps}$ after energy deposition) and SAXS measurements (as-implanted a-Si, 185-MeV Au). The inset shows the time evolution of the radial MD temperature profile (kinetic energy in units of $\mathrm{K}$ ). The dotted lines indicate the melting temperature of as-implanted and relaxed $\mathrm{a}-\mathrm{Si}^{19,20}$ and the corresponding radius of the temperature profile.

resolidification of an ion track may well be sufficient to quench in metastable HDA Si. Alternatively, SAXS determinations of core-shell structures for ion tracks in a-SiO ${ }_{2}^{12}$ or a-Ge ${ }^{13}$ were consistent with an underdense core and overdense shell, which can be attributed to radially outward material flow. ${ }^{13,28}$ While the SAXS experiment cannot unambiguously establish the sign of the density of core and shell, the MD simulations support the notion of ion tracks in a-Si consisting of an overdense core and underdense shell.

At first glance, the results presented above could be perceived as contradictory to the well-known Trinkaus model, ${ }^{29,30}$ given that frozen-in structural remnants of HDL Si should yield a negative relaxation strain and negative deformation yield, with the latter not consistent with experimental measurements of this parameter ${ }^{3}$. However, it is important to note that our low-fluence $\left(<5 \times 10^{11}\right.$ ions $\left./ \mathrm{cm}^{2}\right)$ experiments were designed to minimize ion track overlap. In contrast, a measurable deformation yield requires an incubation fluence ${ }^{31}$ of $\approx 1 \times 10^{12}$ ions $/ \mathrm{cm}^{2}$ and is commonly characterized in the fluence range of $(1-10) \times 10^{14} \mathrm{ions} / \mathrm{cm}^{2}$, where incoming ions are incident on material already subjected to hundreds or thousands of ion impacts. ${ }^{3}$ As a consequence, the material is, overall, modified at the onset of deformation and conceivably compacted when taking into account the net densification in a single ion track. Thus further irradiation cannot necessarily be expected to yield further compaction. This is consistent with SHI irradiation-induced density fluctuations in $\mathrm{SiO}_{2}$ that reach a steady state when the material is fully covered by ion tracks. ${ }^{32}$

\section{CONCLUSIONS}

In conclusion, experimental evidence for ion track formation following swift heavy-ion irradiation of a-Si has been presented. SAXS measurements revealed an underlying coreshell structure in the radial density distribution. MD simulations support the supposition of an overdense core and slightly 
underdense shell compared with the unirradiated a-Si matrix consistent with frozen-in structural remnants of the highdensity liquid phase. The formation of ion tracks was observed in as-implanted a-Si at an electronic energy deposition as low as $10.6 \mathrm{keV} / \mathrm{nm}$, which thus represents an upper limit for the threshold of $S_{e}$ required for ion track formation. Differences in the ion track dimensions between relaxed and as-implanted a-Si have been related to microstructural differences and the increased melting temperature of the former. The observed density fluctuations at the nanoscale in ion tracks in a-Si bring new insights into the early stage of damage formation under high electronic energy deposition and may provide a pathway to stabilize high-density amorphous $\mathrm{Si}$ at ambient temperature and pressure.

\section{ACKNOWLEDGMENTS}

The authors acknowledge the Australian Research Council, the Australian Synchrotron, the IT Center for Science CSC Finland, the supercomputer center NERSC, which is supported by the Office of Science of the US Department of Energy, and the Deutsche Forschungsgemeinschaft for financial support. O.H.P. is supported by the US Department of Energy, Basic Energy Sciences, Materials Science and Engineering Division. Part of the research was undertaken at the SAXS/WAXS beam line of the Australian Synchrotron, Victoria, Australia, the Canberra node of the Australian National Fabrication Facility, and the ANU Heavy-Ion Accelerator Facility (HIAF). We thank the ANU HIAF staff for technical support. *thomas.bierschenk@anu.edu.au

†Present address: Instituto de Física, Universidade Federal do Rio Grande do Sul, C.P. 15051, 91500-970 Porto Alegre, Porto Alegre, Brazil.

${ }^{1}$ M. Toulemonde, C. Dufour, and E. Paumier, Phys. Rev. B 46, 14362 (1992).

${ }^{2}$ I. M. Lifshits, M. I. Kaganov, and L. V. Tanatarov, J. Nucl. Eng., Part A 12, 69 (1960).

${ }^{3}$ A. Hedler, S. L. Klaumünzer, and W. Wesch, Nat. Mater. 3, 804 (2004).

${ }^{4}$ A. Hedler, S. Klaumünzer, and W. Wesch, Nucl. Instrum. Methods Phys. Res., Sect. B 242, 85 (2006).

${ }^{5}$ A. Dunlop, G. Jaskierowicz, and S. Della-Negra, Nucl. Instrum. Methods Phys. Res., Sect. B 146, 302 (1998).

${ }^{6}$ A. Kamarou, W. Wesch, E. Wendler, A. Undisz, and M. Rettenmayr, Phys. Rev. B 78, 054111 (2008).

${ }^{7}$ B. Canut, N. Bonardi, S. Ramos, and S. Della-Negra, Nucl. Instrum. Methods Phys. Res., Sect. B 146, 296 (1998).

${ }^{8}$ A. Chettah, H. Kucal, Z. Wang, M. Kac, A. Meftah, and M. Toulemonde, Nucl. Instrum. Methods Phys. Res., Sect. B 267, 2719 (2009).

${ }^{9}$ S. Furuno, H. Otsu, K. Hojou, and K. Izui, Nucl. Instrum. Methods Phys. Res., Sect. B 107, 223 (1996).

${ }^{10}$ M. Rodrguez, B. Afra, C. Trautmann, M. Toulemonde, T. Bierschenk, J. Leslie, R. Giulian, N. Kirby, and P. Kluth, J. Non-Cryst. Solids 358, 571 (2012).

${ }^{11}$ P. Kluth, C. Schnohr, D. Sprouster, A. Byrne, D. Cookson, and M. Ridgway, Nucl. Instrum. Methods Phys. Res., Sect. B 266, 2994 (2008).

${ }^{12}$ P. Kluth, C. S. Schnohr, O. H. Pakarinen, F. Djurabekova, D. J. Sprouster, R. Giulian, M. C. Ridgway, A. P. Byrne, C. Trautmann, D. J. Cookson, K. Nordlund, and M. Toulemonde, Phys. Rev. Lett. 101, 175503 (2008).

${ }^{13}$ M. C. Ridgway, T. Bierschenk, R. Giulian, B. Afra, M. D. Rodriguez, L. L. Araujo, A. P. Byrne, N. Kirby, O. H. Pakarinen, F. Djurabekova, K. Nordlund, M. Schleberger, O. Osmani, N. Medvedev, B. Rethfeld, and P. Kluth, Phys. Rev. Lett. 110, 245502 (2013).
${ }^{14}$ B. Haberl, A. C. Y. Liu, J. E. Bradby, S. Ruffell, J. S. Williams, and P. Munroe, Phys. Rev. B 79, 155209 (2009).

${ }^{15}$ J. Ziegler, J. Biersack, and M. Ziegler, http://www.srim.org.

${ }^{16}$ B. Afra, M. Rodriguez, M. Lang, R. Ewing, N. Kirby, C. Trautmann, and P. Kluth, Nucl. Instrum. Methods Phys. Res., Sect. B 286, 243 (2012).

${ }^{17}$ J. S. Custer, M. O. Thompson, D. C. Jacobson, J. M. Poate, S. Roorda, W. C. Sinke, and F. Spaepen, Appl. Phys. Lett. 64, 437 (1994).

${ }^{18}$ S. Roorda, W. C. Sinke, J. M. Poate, D. C. Jacobson, S. Dierker, B. S. Dennis, D. J. Eaglesham, F. Spaepen, and P. Fuoss, Phys. Rev. B 44, 3702 (1991).

${ }^{19}$ M. G. Grimaldi, P. Baeri, M. A. Malvezzi, and C. Sirtori, Int. J. Thermophys. 13, 141 (1992).

${ }^{20}$ M. G. Grimaldi, P. Baeri, and M. A. Malvezzi, Phys. Rev. B 44, 1546 (1991).

${ }^{21}$ K. Nordlund, M. Ghaly, R. S. Averback, M. Caturla, T. Diaz de la Rubia, and J. Tarus, Phys. Rev. B 57, 7556 (1998).

${ }^{22}$ J. Tersoff, Phys. Rev. B 38, 9902 (1988).

${ }^{23}$ F. Wooten, K. Winer, and D. Weaire, Phys. Rev. Lett. 54, 1392 (1985).

${ }^{24}$ H. J. C. Berendsen, J. P. M. Postma, W. F. van Gunsteren, A. DiNola, and J. R. Haak, J. Chem. Phys. 81, 3684 (1984).

${ }^{25}$ M. Beye, F. Sorgenfrei, W. F. Schlotter, W. Wurth, and A. Föhlisch, Proc. Natl. Acad. Sci. USA 107, 16772 (2010).

${ }^{26}$ F. H. Stillinger and T. A. Weber, Phys. Rev. B 31, 5262 (1985).

${ }^{27}$ P. F. McMillan, M. Wilson, D. Daisenberger, and D. Machon, Nat. Mater. 4, 680 (2005).

${ }^{28}$ K. Gärtner, J. Jöhrens, T. Steinbach, C. S. Schnohr, M. C. Ridgway, and W. Wesch, Phys. Rev. B 83, 224106 (2011).

${ }^{29}$ H. Trinkaus and A. I. Ryazanov, Phys. Rev. Lett. 74, 5072 (1995).

${ }^{30}$ H. Trinkaus, Nucl. Instrum. Methods Phys. Res., Sect. B 146, 204 (1998).

${ }^{31}$ S. Klaumünzer, M.-D. Hou, and G. Schumacher, Phys. Rev. Lett. 57, 850 (1986).

${ }^{32}$ P. Kluth, O. H. Pakarinen, F. Djurabekova, R. Giulian, M. C. Ridgway, A. P. Byrne, and K. Nordlund, J. Appl. Phys. 110, 123520 (2011). 